\title{
New Results on Testing Duality in Spin Structure from Jefferson Lab
}

\author{
Nilanga Liyanage \\ University of Virginia, Charlottesville, VA, USA
}

\begin{abstract}
The Bloom-Gilman duality has been experimentally demonstrated for spin independent structure functions. Duality is observed when the smooth scaling curve at high momentum transfer is an average over the resonance bumps at lower momentum transfer, but at the same value of scaling variable $x$. Signs of quark-hadron duality for the spin Dependant structure function $g_{1}$ of the proton has been recently reported by the Hermes collaboration. Experimental Halls A, B and $\mathrm{C}$ at Jefferson lab have recently measured spin structure functions in the resonance region for the proton and the neutron. Data from these experiments combined with Deep-Inelastic-Scattering data provide a precision test of quark-hadron duality predictions for spin structure functions for both the proton and the neutron. This will be one of the first precision tests of spin and flavor dependence of quark-hadron duality.
\end{abstract}

Keywords: Quark-hadron duality, nucleon, spin

Thirty years ago Bloom and Gilman [1] made the observation that the scaling curve seen at high momentum transfer is an accurate average over the resonance bumps at lower momentum transfer but at the same value of $x$. This duality between the resonance region, which is best described by constituent quark models, and the scaling region, which is well described by $\mathrm{pQCD}$, hints a common origin for both regions. Recent data from Jefferson Lab Hall C [2] have further confirmed that Bloom-Gilman duality holds to a few percent level down to small values of $Q^{2}$. The striking agreement shown by these data for unpolarized structure functions between the resonance and the scaling regions raises the question whether duality holds for polarized structure functions as well. Hermes collaboration has recently reported observing global quark-hadron duality for the spin structure function $g_{1}$ of the proton [3]. For a review of the published duality data and theoretical models, see [4].

Recently Jefferson lab Experimental Halls A, B and C have conducted precision experiments to measure spin structure functions in the resonance region for both the proton and the neutron (polarized ${ }^{3} \mathrm{He}$ and deuteron).

Jefferson Lab Hall A experiment E01-012 used Hall A polarized ${ }^{3} \mathrm{He}$ target for a precision extraction of the neutron spin structure functions $g_{1}^{n}, g_{2}^{n}$ and the virtual photon asymmetries $A_{1}^{n}, A_{2}^{n}$ in the resonance region up to $Q^{2}=4(\mathrm{GeV} / \mathrm{c})^{2}$. Both Hall A High Resolution spectrometers (HRS) were used in a symmetric configuration in electron detection mode to measure the inclusive ${ }^{3} \vec{H} e\left(e, e^{\prime}\right) X$ reaction. Three beam energies, $3 \mathrm{GeV}, 4 \mathrm{GeV}$ and $5 \mathrm{GeV}$ were used with spectrometer angles of $25^{\circ}$ and $32^{\circ}$. At each kinematic setting parallel and perpendicular cross sections and asymmetries were measured with the target spin parallel and perpendicular to the electron beam respectively. 

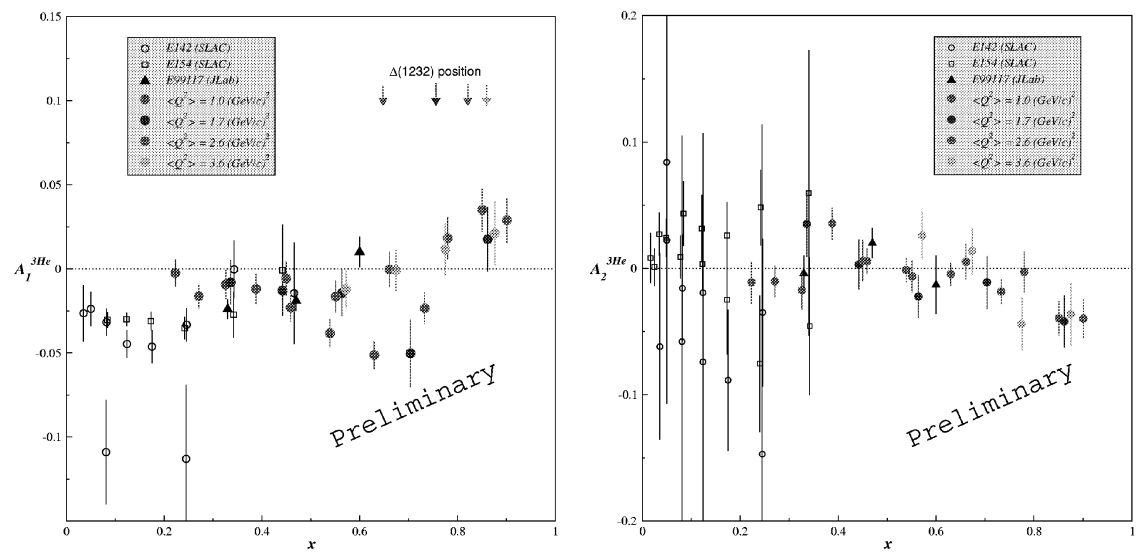

FIGURE 1. Preliminary results from Hall A experiment E01-012 for $A_{1}^{3} \mathrm{He}$ (left) and $A_{2}^{3} \mathrm{He}$ (right), compared $A_{1}^{3} \mathrm{He}$ and $A_{2}^{3} \mathrm{He}$ measured in the DIS region from Hall A experiment E99-117. The arrow indicates the location of the $\Delta(1232)$ resonance.

Figure 1 (left) shows preliminary results for $A_{1}^{3} \mathrm{He}$ in the resonance region at the four $Q^{2}$ values, compared to $A_{1}^{3} \mathrm{He}$ in the DIS region from Hall A experiment E99-117 [5]. The position of the $\Delta(1232)$ resonance is indicated by an arrow. The error bars shown are statistical only. The most noticeable feature of the plot is the negative contribution due to $\Delta(1232)$ at the two low $Q^{2}$ settings $\left(Q^{2}<2(\mathrm{GeV} / \mathrm{c})^{2}\right)$. It has been noted that quarkhadron duality for spin structure functions is not expected in the Delta resonance region at this low $Q^{2}$. For the two higher $Q^{2}$ settings, $A_{1}^{3} H e$ at the location of $\Delta(1232)$ is positive. It is also interesting to note that the results from these two settings $\left(Q^{2}>2(\mathrm{GeV} / \mathrm{c})^{2}\right)$ agree perfectly with each other, showing little or no $Q^{2}$ dependence, as expected in the scaling region. Furthermore, Our data seem to indicate that with increasing $x_{B j}, A_{1}$ goes from negative to positive showing the same trend as indicated by the DIS data from Experiment 99-117. The behavior of $A_{1}^{n}$ becoming positive at high $x_{B j}$ has been predicted for DIS data by relativistic constituent quark models and by pQCD inspired models [6].

Jefferson Lab Hall B CLAS collaboration's EG1 measured polarized structure functions for the proton and the deuteron in the resonance region covering a kinematic range of $0.05<Q^{2}<4.5(\mathrm{GeV} / \mathrm{c})^{2}$ and $0.2<x<0.8$. Hall B Longitudinally polarized ammonia targets were used for this experiment and the scattered electrons were detected in the CEBAF Large Acceptance Spectrometer (CLAS). A detailed description of the experimental setup can be found in references [7,8]. The preliminary results for $g_{1}^{p}$ are shown in Figure 2 compared to a parametrization of world's DIS data at $Q^{2}=10(\mathrm{GeV} / \mathrm{c})^{2}$. For the settings where $Q^{2}<2(\mathrm{GeV} / \mathrm{c})^{2}, g_{1}^{p}$ is negative at the $\Delta(1232)$ resonance, clearly deviating from the DIS parametrization. However, at the higher $Q^{2}$ settings resonance data 
appear to be in closer agreement with the DIS parametrization indicating the onset of quark-hardron duality.

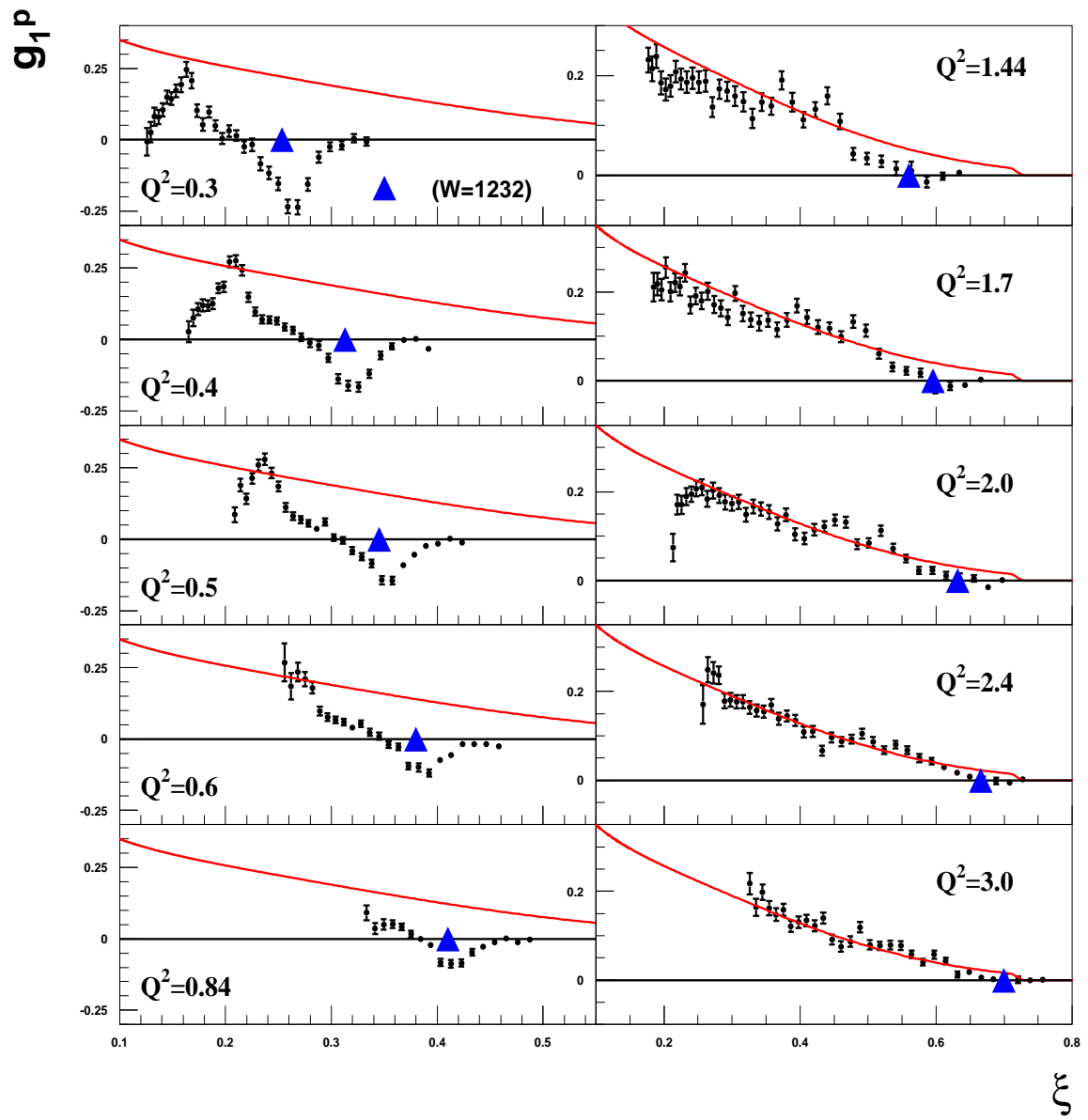

FIGURE 2. Preliminary results for $g_{1}^{p}$ from Hall B EG1b experiment. The lines represent a parametrization of world's DIS data at $Q^{2}=10(\mathrm{GeV} / \mathrm{c})^{2}$. The arrow indicates the location of the $\Delta(1232)$ resonance.

Jefferson lab Hall C experiment E01-006, Resonance Spin Structure (RSS), preformed a precision measurement of spin asymmetries $A_{1}$ and $A_{2}$ and spin structure functions $g_{1}$ and $g_{2}$ for the proton and the deuteron at $Q^{2} \approx 1.3(\mathrm{GeV} / \mathrm{c})^{2}$ and $0.8<W<$ $2 \mathrm{GeV}$. This experiment used the UVa-Jefferson lab polarized ammonia target which allowed the measurement of both parallel and transverse asymmetries. The scattered electrons were detected in the Hall C High Momentum Spectrometer (HMS). Figure 3 shows preliminary results for $g_{1}$ and $g_{2}$ for the proton. The $g_{1}^{p}$ results, measured at $Q^{2}=1.3(\mathrm{GeV} / \mathrm{c})^{2}$, are compared to a parametrization of $g_{1}^{p}$ in the DIS region evaluated at $Q^{2}=1.3(\mathrm{GeV} / \mathrm{c})^{2}$. At the $\Delta(1232)$ resonance $g_{1}^{p}$ is negative, dipping well below the DIS scaling curve, while at the higher resonance regions the resonance data appear to oscillate around the scaling curve. These observations are in qualitative agreement with 

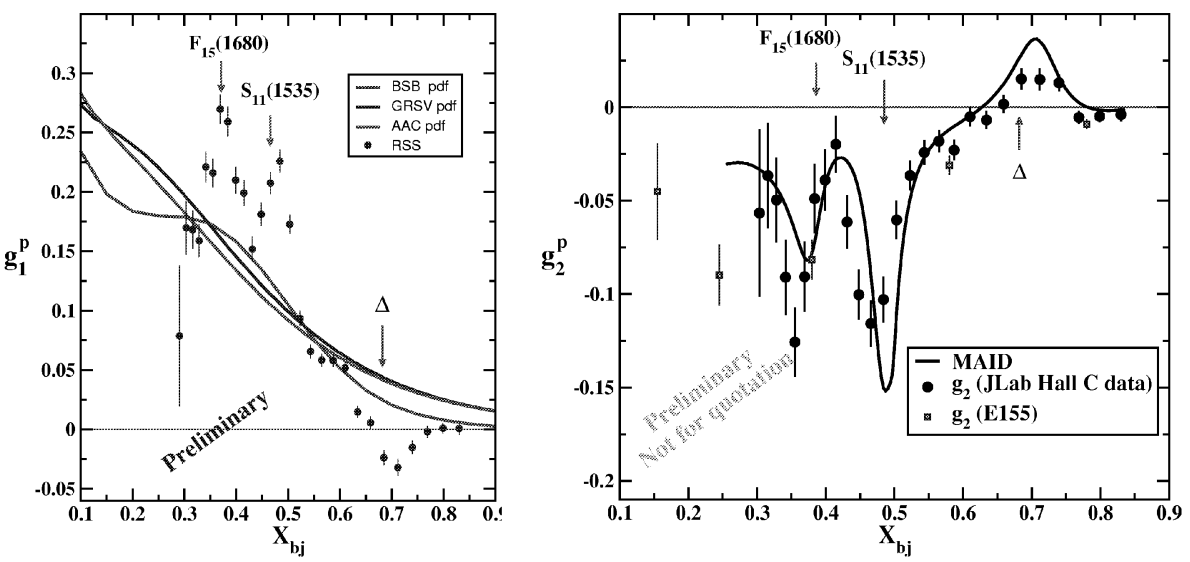

FIGURE 3. Preliminary results for $g_{1}^{p}$ and $g_{2}^{p}$ from Hall C RSS experiment. The lines on the $g_{1}^{p}$ plot represent parameterizations of world's DIS data at $Q^{2}=1.3(\mathrm{GeV} / \mathrm{c})^{2}$. The line shown on the $g_{2}^{p}$ is from the MAID model.

Hall A and Hall B results taken at $Q^{2}<2(\mathrm{GeV} / \mathrm{c})^{2}$ settings.

Summary and Outlook

Polarized experiments in Jefferson lab Halls A, B and C provide precision spin structure data in the resonance region for both the proton and the neutron. These data will provide a comprehensive test of the spin and flavor dependence of quark hadron duality. Preliminary results from all three experiments indicate that for $Q^{2}$ less than approximately $1.5(\mathrm{GeV} / \mathrm{c})^{2}$, quark-hadron duality is violated for $g_{1}$ structure function, mostly due to the strong negative contribution of $\Delta(1232)$ resonance. Data taken at $Q^{2}>2(\mathrm{GeV} / \mathrm{C})^{2}$ provide qualitative indications of global quark-hadron duality for $g_{1}$ and $A_{1}$.

The data analysis of all three experiments are in the final stages. Quantitative tests of global and local quark-hadron duality are being carried out with these data. Final results are expected soon.

\section{REFERENCES}

1. E. D. Bloom and F. J. Gilman, Phys. Rev. Lett. 25, 1140 (1970); Phys. Rev. D 4, 2901 (1971).

2. I. Niculescu et al., Phys. Rev. Lett. 85, 1186 (2000); I. Niculescu et al., Phys. Rev. Lett. 85, 1182 (2000).

3. A. Airapetian et al., Phys. Rev. Lett. 90,092002 (2003).

4. W. Melnitchouk, R. Ent and C. Keppel, Phys.Rept. 406, 127-301 (2005).

5. X. Zheng et al., Phys. Rev. C 70, 065207 (2004); X. Zheng et al., Phys. Rev. Lett. 92,012004 (2004).

6. See the discussion in X. Zheng et al., Phys. Rev. C 70 and references therein.

7. Vipuli Dharmawardane, Ph.D Thesis, Old Dominion University (2004)

8. Yelena Prok, Ph.D Thesis, University of Virginia (2004). 